International Journal of Advanced Trends in Computer Science and Engineering

Available Online at http://www.warse.org/IJATCSE/static/pdf/file/ijatcse73942020.pdf

https://doi.org/10.30534/ijatcse/2020/73942020

\title{
Data-Driven Methods for Next Generation of Wireless Communication Networks
}

\author{
Vijayakumar Ponnusamy ${ }^{1}$, T. Karthick ${ }^{2}$, R. Nandakumar ${ }^{3}$, Yuvaraj $^{4}$ \\ ${ }^{1, A}$ Associate Professor, Dept of ECE, SRMIST, Chennnai, vijayakp@ srmist.edu.in \\ ${ }^{2}$ Assistant Professor, Dept of IT, SRMIST, Chennnai, karthict@ srmist.edu.in \\ ${ }^{3}$ Professor \& Head, Department ECE, K S R Institute for Engineering and Technology, Tiruchengode \\ ${ }^{4}$ Assistant Professor ,Dept of ECE, SRMIST, Chennnai, yuvasivasanthi@ gmail.com
}

\begin{abstract}
The next generation of wireless communications networks will be very complex with the heterogeneous nature of nodes, radio access mechanisms, communication links, and network slices. The network will be more dynamic, which requires an intelligent system to address those complex heterogeneous scenarios and to adapt to the dynamics of wireless channels and environments. Machine Learning (ML) and Deep Learning (DL)-based solutions will be the optimal one for this problem. Machine learning and Deep learning driven algorithms can facilitate to analyze and make efficient resource management.
\end{abstract}

Key words : data-driven method, deep learning, end-to-end Learning ,machine learning, wireless communication

\section{INTRODUCTION}

Cognitive RADIO (CR) is used to understand and respond to society through the use of radios. The growing demand for wireless bandwidth in the radio spectrum plays a significant part in automatic signal detection and modulation recognition. The modulation model and estimated signal parameters within the operating streams will thus be specified by users and can help reconfigure a link and calculate the electromagnetic environment. It is also widely used both in military and civil applications that have attracted a lot of attention decades ago. The identification of multi-signals is a function to locate current signals within particular broadband, one of the critical components of CR. Energy is an essential difference between signal and non-signal. As a consequence, several broadband algorithms focused on the power detector (ED) multi-signal identification.

Machine learning, soft computing, and deep learning mechanisms are applied to wireless communication for improved efficiency [19-22]. Excellent anti-noise efficiency is accomplished by high-order, statistically dependent algorithms, such as signal high-order cumulative and cyclic continuum. These methods have a relatively low computational complexity, but the selection of features relates too much to experienced experts. Attributes that can be adjusted to non-ideal environments are challenging to acquire. Especially as several modulation formats need to be categorized, it is difficult to set the decision threshold.

Deep Learning ( DL) has made remarkable milestones with computer vision (CV) and Natural Language Processing ( NLP) due to its high ability to understand itself. Increasing numbers of scholars have used DL methods to deal with signal processing issues. Throughout the narrowband method, several DL-based signal recognition strategies were used. These methods only detect signals and thus can not calculate associated parameters. It is a daunting challenge to devise a technique that draws on profound information to distinguish signals consistently and effectively.

The use cases/applications of the machine and deep learning for wireless communication are summarized below

A) Signal Detection/Classification Using ML/DL

B) End-to-End Learning of Communications Systems

C)Channel Estimation Using ML/DL

D)Resource Allocation Using ML/DL

The popularity of the wireless program DL is attributed to three correlations between DL and the human intellect. 1. Incomplete or even wrong data entry resistance, 2 . Ability to handle a large number of inputs: 3 . The freedom to rely on decisions

\section{SIGNAL DETECTION/CLASSIFICATION USING ML/DL}

The trained deep neural network takes less time and ability to provide reliable signal detection as compared to traditional detection mechanism, which involves the iterative, algorithmic signal sample search, and detection [12]. In wireless networks, DL plays a significant role in the physical layer (PL). For example, due to the thorough study of the dynamic radio conditions involving exposure to the spectrum, interference propagation, node stability, device types, etc., the DL may help decide the most effective modulation/encoding schemes.

Interference coordination and jamming resistance in wireless networks are two of the most challenging problems in consideration of the vast number of routers, node stability, channel state variation, and dynamical use of frequencies. DL is the best method for dealing with dynamic challenges 
because, through its underlying nature, DL overrides intermediate and broad physical layer parameters. In the context of modern systems such as orthogonal multiplexing frequency splitting (OFDM), the modulation and correction of errors are essential physical layer functions that require enormous computational computations. DL methodology could dramatically increase the efficiency of these operations. Nevertheless, the majority of the physical layer issues have rigid reaction time constraints, and the use of the DL system and the management of device complexity are essential questions for physical layer DL implementations.

\subsection{DL for Interference Alignment}

The cache-enables opportunistic IA networks use profound Q-learning to achieve the optimum IA user selection strategy, as seen in Figure 1. A central scheduler here collects each user's channel status and cache status, as well as assigning channel resources to each user. It is the time-varying channel defined by the Markov model of the Finite State. Each transmitter has a cache that stores a lot of information that is often required. For this in-network architecture, redundant information is minimized effectively.

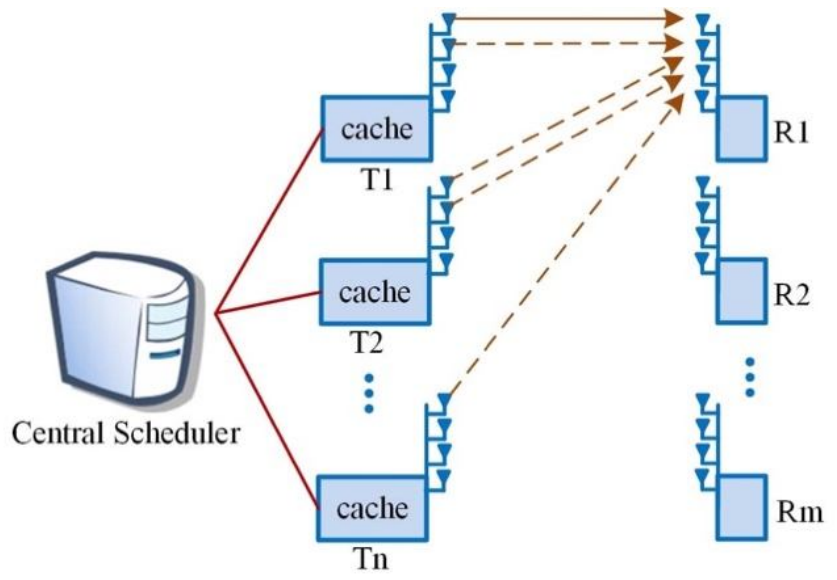

Figure 1: Cache-Enabled Opportunistic IA Networks.

\subsection{DL for Jamming Resistance}

A two-dimensional, profound Q-learning method for Secondary User (SU) anti-jamming. It uses both frequency hopping and SU versatility to meet intelligent jammers, as shown in Figure 2. Furthermore, whether the SU is going is not explicitly discussed. To find the right access point and to avoid repeat requests, a successful SU moving strategy is necessary.

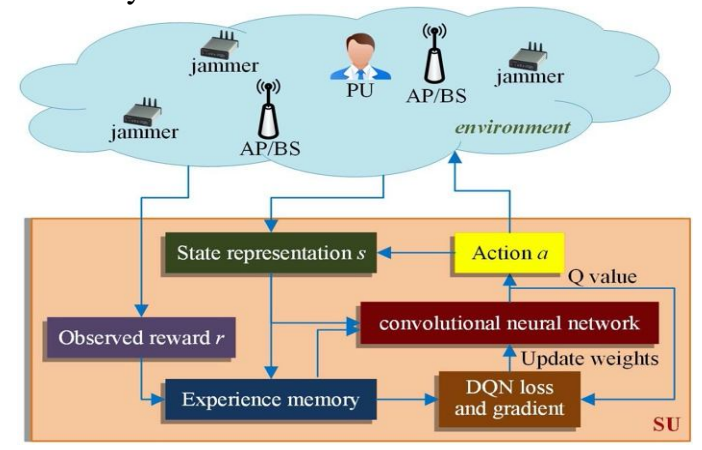

Figure 2: DQN-based Anti-Jamming System.

\section{END-TO-END LEARNING OF COMMUNICATIONS SYSTEMS}

End-to-end communication systems are a fascinating new technology whose aim is to learn the full installation of transmitters and receivers, which can be designed for a particular channel and measurement output model. The system can be defined as Neural Networks (NNs), and the whole system can be viewed as an autoencoder[1-4]. This can be achieved.

- Advantage: Hopefully, no mathematical channel model is needed so that it can be implemented without preliminary testing to any form of channel.

- Benefit: performance varies with parameters to change Feature Learning.

Application learning is focused instead of building expert applications on raw data and sampling data. The CNN classifier [10] is a newly introduced AMC scheme based on the DL concept. The IQ time-domain samples are fed directly to the CNN model, and correct matched filters in the various SNRs can be learned automatically. CNN systems of varying sizes and depths have been tested for classification accuracy. The authors have presented a hybrid learning system, incorporating $\mathrm{CNN}$ and long-term memory (LSTM) to enhance classification efficiency.

Simulation and real signals are reported as having a significant effect on the precision of the frequency offset and noise. In comparison [13], a novel model has been proposed to achieve the frequency correction approximation and phase noise to increase accuracy efficiency. The authors introduce a Signal Distortion Module (CM) to boost further the efficiency of CNN-based reconnaissance schemes in [10], and the results indicate that this $\mathrm{CM}+\mathrm{CNN}$ scheme is more reliable than current schemes. The HDMF solution is suggested in $[14,15]$, and the application of CNN and LSTM is addressed without any prior knowledge. There are no prior details available. Without external operations, the time domain data is transmitted to the fusion device. The findings show that HDMF is an outstanding solution to heterogeneous networks with much higher efficiency.

\section{CHANNEL ESTIMATION USING ML/DL}

The next-generation wireless network is slated to support a large number of connected devices with extremely high data rates, thus paving the way for massive MIMO.

The channel estimation becomes extremely important at the same time very challenging because of a large number of connected devices and a large number of service antennas at the base station. General orthogonal pilot-based channel estimation (CE) schemes and other existing estimation schemes will result in an excessive amount of overhead. ML/DL, which can solve very complex and non-convex problems with ease, is expected to address this issue of channel estimation in the next-generation wireless network. 
Channel estimation is done by machine and deeplerning[5-7]. A comprehensive analysis (DL), Basic Multi-Input Multi-Output (MIMO) Massive Channel Estimate Scheme was suggested by Chang-Jae Chun[8]. Contrary to current research, in case the pilot length is higher than the number of transmission antennas, we establish a channel approximation system. The proposed scheme involves two phases: a DL pilot-assisted channel estimate and a DL data-driven channel estimate. Within the first step, a two-layer neural network (TNN) and a deep neural network (DNN) are built to co-develop the pilots themselves and the channel estimators. In the second stage, the precision of the estimation of the channel is further increased by the iterative use of a different DNN. The results of the simulation show that the channel estimate scheme proposed has significantly better results than traditional channel estimates. Due to the number of transmission antennas, and also receive valuable details on the optimum pilot range. By using the neural networks, we build a DL-based channel estimation system. The structure of the proposed DL channel estimate system the pilot-assisted estimation of the DL channel and DL data iterative estimation of the channel

\section{RESOURCE ALLOCATION USING ML/DL}

Potential telecommunications networks would address environments, such as ultra-dense suburban areas and office buildings, subways, roads, and high-speed trains with respect to high-speed travel, incredibly high link capacity, and ultra-fast mobility. Because of its ability to guarantee user quality $(\mathrm{QoS})$ and optimize the use of services to maximize revenues for operators, it is always a hot topic for future wireless communications to allocate radio resources more efficiently[9,11]. The allocation of resources is an issue of non-convex optimization, but the latest research indicates a solution strategy. Power control, spectrum control, backhaul control, cache management, and beamformer software also require the regulation of resources.

The proposed heterogeneous network (HetNet). The cellular explosion of upcoming fifth-generation network $(5 \mathrm{G})$ is a potential option for treating. In this article, HetNets is designed to optimize energy efficiency for a specific subchannel and power distribution problem. A Convolutional Neural Network (CNN) method is built to achieve decisions about sub-channel and power distribution much less complicated than usual by broken down the original problem into a sub-classification and regression sub-problem. Further empirical tests indicate that the suggested $\mathrm{CNN}$ is equal to the exhaustive method, while it executes with $6.76 \%$ of its CPU runtime.

This work will summarize critical contributions as follows.

- With the OFDM HetNet in mind, we devise the assignment of resources as a common subchannel and power issue, which maximizes the network's energy efficiency ( EE) while meeting the spectrum efficiency (SE) requirement.

- Unlike [16]-[18], the proposed C NN decomposes the original issue into a sub-classification problem and sub-problem of regression, to assume an energy efficiency judgment on the joint sub-channel and power allocation, and address both regression and resource distribution problems by deep learning.

- A variety of detailed studies are performed to demonstrate that the CNN proposal will work similarly to the Thorough process while reducing processing time substantially.

The solution suggested splits the original problem into a sorting problem and a regression question and generates energy-efficient decisions with low numerical complexity on the joint subchannel and resource allocation.

\section{SYSTEM MODEL FOR DATA-DRIVEN METHODS FOR NEXT GENERATION OF WIRELESS COMMUNICATION NETWORKS}

Three wide classes are categorized under machine learning systems part of the Artificial Intelligence Systems: supervised testing, non-supervised learning, and augmented learning. Networks, state vector (SVM), hidden Markov sequences (learning under supervision) are used as the essential techniques of semantic radio networks. According to research performed with the use of cognitive radio algorithms, two classes may be divided: the "classification logical" and the "decision-making," which are considered to have been applied.

A type definition is mainly related to spectral and signal processing. "Engineering" means adaptive modulation, signal strength control, routing, and transportation-layer goals. The natural modeling approach, theoretically inherent, is common in the study of the functioning of the neural network using radio access, in addition to mathematical modeling techniques.

Within this study, we address many learning algorithms that CRs can use to accomplish various goals. To order to get a deeper understanding of the functions and differences between the algorithms presented, we define two key problem groups and demonstrate the learning algorithms for each category. The hierarchical structure and dependency of learning algorithms are shown in Figure. 3. The reference to Figure. 3, we define two major CR problems (or tasks) as follows:

- Decision-making.

- Classification of features. 
Such issues are common in the sense that they involve a wide variety of CR functions. For example, classification problems occur in spectrum sensing, while decision-making issues emerge in the determination of spectrum sensing, power management, or adaptive modulation.Decisison making maily used fro channel selection[23]. There are various feature selection[24] which can be used for this purpose .

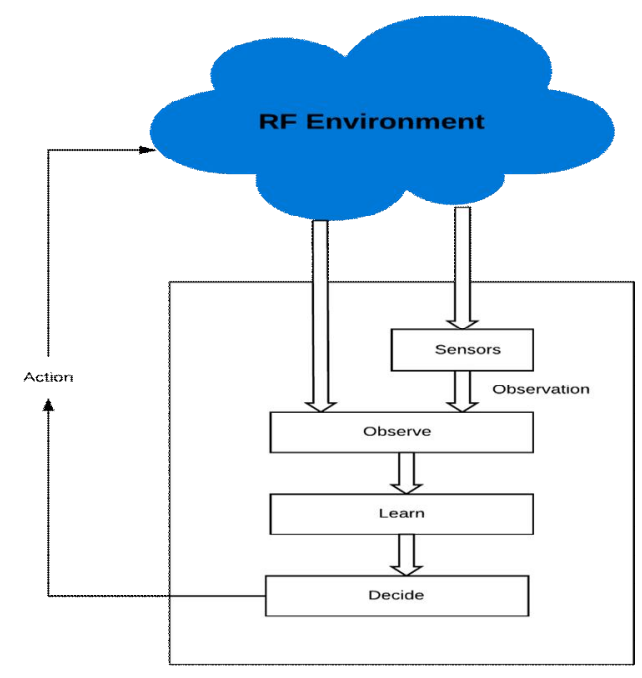

Figure 3: System Model for Data-Driven Methods for Next Generation of Wireless Communication Networks

\section{CONCLUSION}

In this survey paper, we described the learning problems in $\mathrm{CRs}$ and the significance of machine learning in the development of actual CRs. They introduced state-of-the-art research approaches applicable to CRs classifying them as supervised and unsupervised research. Analysis of some of the most important and widely used learning algorithms have been presented along with their advantages and drawbacks. We also discussed some of the problematic learning challenges faced in CRs and introduced potential solutions to them. The network would be more diverse, needing an adaptive device to handle these complex heterogeneous situations and respond to the complexities of wireless networks and ecosystems. Machine Learning (ML) and Deep Learning (DL)-based approaches would be the ideal approach to this problem. Machine learning and deep learning algorithms can promote research and effective resource management.

\section{REFERENCES}

1. Wu, N., Wang, X., Lin, B., and Zhang, K., 2019. A CNN-Based End-to-End Learning Framework Toward Intelligent Communication Systems. IEEE Access, 7, pp.110197-110204. https://doi.org/10.1109/ACCESS.2019.2926843

2. Aoudia, F.A. and Hoydis, J., 2018, October. End-to-end learning of communications systems without a channel model. In 2018 52nd Asilomar Conference on Signals, Systems, and Computers (pp. 298-303). IEEE.

3. Aoudia, F.A. and Hoydis, J., 2019. Model-free training of end-to-end communication systems. IEEE Journal on Selected Areas in Communications, 37(11), pp.2503-2516.

4. Ye, H., Li, G.Y., Juang, B.H.F. and Sivanesan, K., 2018, December. Channel agnostic end-to-end learning based communication systems with conditional GAN. In 2018 IEEE Globecom Workshops (GC Wkshps) (pp. 1-5). IEEE.

5. Yuan, J., Ngo, H.Q. and Matthaiou, M., 2019, July. Machine Learning-Based Channel Estimation in Massive MIMO with Channel Aging. In 2019 IEEE 20th International Workshop on Signal Processing Advances in Wireless Communications (SPAWC) (pp. 1-5). IEEE.

6. Motade, S.N. and Kulkarni, A.V., 2018. Channel estimation and data detection using machine learning for MIMO 5G communication systems in fading channel. Technologies, 6(3), p.72.

https://doi.org/10.3390/technologies6030072

7. Chun, C.J., Kang, J.M. and Kim, I.M., 2019. Deep learning-based channel estimation for massive MIMO systems. IEEE Wireless Communications Letters, 8(4), pp.1228-1231.

8. He, H., Wen, C.K., Jin, S. and Li, G.Y., 2018. Deep learning-based channel estimation for beamspace mmWave massive MIMO systems. IEEE Wireless Communications Letters, 7(5), pp.852-855.

9. Wang, J.B., Wang, J., Wu, Y., Wang, J.Y., Zhu, H., Lin, M. and Wang, J., 2018. A machine learning framework for resource allocation assisted by cloud computing. IEEE Network, 32(2), pp.144-151.

10. Ahmed, I. and Khammari, H., 2018, December. Joint machine learning based resource allocation and hybrid beamforming design for massive mimo systems. In 2018 IEEE Globecom Workshops (GC Wkshps) (pp. 1-6). IEEE.

11. Xu, D., Chen, X., Wu, C., Zhang, S., Xu, S. and Cao, S., 2019, April. Energy-efficient subchannel and power allocation for hetnets based on convolutional neural network. In 2019 IEEE 89th Vehicular Technology Conference (VTC2019Spring) (pp. 1-5). IEEE

12. Zha, Xiong, Hua Peng, Xin Qin, Guang Li, and Sihan Yang. "A Deep Learning Framework for Signal Detection and Modulation Classification." Sensors 19, no. 18 (2019): 4042.

13. K. Yashashwi, A. Sethi, and P. Chaporkar, "A learnable distortion correction module for modulation recognition," IEEE Wireless Communications Letters, pp. 1-1, 2018. https://doi.org/10.1109/LWC.2018.2855749 
14. D. Zhang, W. Ding, B. Zhang et al., "Automatic modulation classification based on deep learning for unmanned aerial vehicles," Sensors, vol. 18, no. 3, p. 924, 2018.

15. D. Zhang, W. Ding, B. Zhang et al., Heterogeneous Deep Model Fusion for Automatic Modulation Classification, 2018.

16. H. Sun, X. Chen, Q. Shi, M. Hong, X. Fu, and N. D. Sidiropoulos, "Learning to optimize: Training deep neural networks for wireless resource management," in IEEE International Workshop on Signal Processing Advances in Wireless Communications, 2017, pp. 1-6.

17. W. Lee, M. Kim, and D. H. Cho, "Deep power control: Transmit power control scheme based on convolutional neural network," IEEE Communications Letters, vol. PP, no. 99, pp. 1-1, 2018.

18. J. B. Wang, J. Wang, Y. Wu, J. Y. Wang, H. Zhu, M. Lin, and J. Wang, "A machine learning framework for resource allocation assisted by cloud computing," IEEE Network, vol. 32, no. 2, pp. 144-151, 2018.

19. P. Vijayakumar, S. Malarvizhi, "Wide Band Full Duplex Spectrum Sensing with Self-Interference Cancellation-an Efficient SDR Implementation "Mobile Networks \& Applications, 22(4), 702-711,2017.

20. P.Vijayakumar, S.Malarvizhi, "Fuzzy Logic Based Decision System for Context Aware Cognitive Waveform Generation", Wireless Personal Communications (ISSN No.0929-6212), 94(4), pp. 2681-2703, 2017.

21. P. Vijayakumar,S. Malarvizhi , "Hardware Impairment Detection and Prewhitening on MIMO Precoder for Spectrum Sharing", Wireless Personal Communication(ISSN No.0929-6212) , Springer Journal, 96(1), pp.1557-1576,2017.

22. P.Vijayakumar, Bahraini Jayapandian Kasthuri and Mayuri.B.R, "Game Theory based Hybrid Cognitive Radio ,Indian Journal of Science and Technology 9 (20),2016 .

23. B. Muni Lavanya 1 C Shoba Bindu2, Fuzzy Logic based Channel Selection Algorithm for Wireless Mesh Networks, International Journal of Advanced Trends in Computer Science and Engineering,8(5),2003-2014,2019. https://doi.org/10.30534/ijatcse/2019/25852019

24. N Chandra Sekhar Reddy1, Dr. Purna Chandra Rao Vemuri2, Dr. A Govardhan3, An Implementation of Novel Feature Subset Selection Algorithm for IDS in Mobile Networks, 8(5),2003-2141,2019 